\title{
On a conjecture about the ratio of Wiener index in iterated line graphs*
}

\author{
Katarína Hriňáková , Martin Knor \\ Faculty of Civil Engineering, Slovak University of Technology, \\ Radlinského 11, 810 05, Bratislava, Slovakia \\ Riste Škrekovski \\ FMF, University of Ljubljana, 1000 Ljubljana and \\ Faculty of Information Studies, 8000 Novo Mesto and \\ FAMNIT, University of Primorska, 6000 Koper, Slovenia
}

Received 9 January 2017, accepted 30 May 2018, published online 23 July 2018

\begin{abstract}
Let $G$ be a graph. Denote by $W(G)$ its Wiener index and denote by $L^{i}(G)$ its $i$ iterated line graph. Dobrynin and Mel'nikov proposed to estimate the extremal values for the ratio $R_{k}(G)=W\left(L^{k}(G)\right) / W(G)$ for $k \geq 1$. Motivated by this we study the ratio for higher $k$ 's. We prove that among all trees on $n$ vertices the path $P_{n}$ has the smallest value of this ratio for $k \geq 3$. We conjecture that this holds also for $k=2$, and even more, for the class of all connected graphs on $n$ vertices. Moreover, we conjecture that the maximum value of the ratio is obtained for the complete graph.
\end{abstract}

Keywords: Wiener index, line graph, tree, iterated line graph.

Math. Subj. Class.: 05C12, 05C05, 05C76

\section{Introduction}

Let $G$ be a graph. We denote its vertex set and edge set by $V(G)$ and $E(G)$, respectively. For any two vertices $u, v$ let $d(u, v)$ be the distance from $u$ to $v$. The Wiener index of $G$, $W(G)$, is defined as

$$
W(G)=\sum_{u \neq v} d(u, v),
$$

* The first and second author acknowledge partial support by Slovak research grants VEGA 1/0007/14, VEGA 1/0026/16, VEGA 1/0142/17, APVV-0136-12 and APVV-15-0220. The research was partially supported by Slovenian research agency ARRS, program no. P1-0383.

E-mail address: hrinakova@math.sk (Katarína Hriňáková), knor@math.sk (Martin Knor), skrekovski@gmail.com (Riste Škrekovski) 
where the sum is taken over all unordered pairs of vertices of $G$. Wiener index was introduced by Wiener in [17]. Since it is related to several properties of molecules (see [7]), it is widely studied by chemists. The interest of mathematicians was attracted in 1970's, when it was reintroduced as the transmission and the distance of a graph, see [16] and [5], respectively. For surveys and some up-to-date papers related to the Wiener index of trees and line graphs see $[15,18]$ and $[2,8,13]$, respectively.

By definition, if $G$ has a unique vertex, then $W(G)=0$. In this case, we say that the graph $G$ is trivial.

The line graph of $G, L(G)$, has vertex set identical with the set of edges of $G$ and two vertices of $L(G)$ are adjacent if and only if the corresponding edges are incident in $G$. Iterated line graphs are defined inductively as follows:

$$
L^{i}(G)= \begin{cases}G & \text { if } i=0 \\ L\left(L^{i-1}(G)\right) & \text { if } i>0\end{cases}
$$

Observe that $W\left(P_{n}\right)=((n-1)+\cdots+1)+((n-2)+\cdots+1)+\cdots+1=\left(\begin{array}{c}n+1 \\ 3\end{array}\right)$. In the case when a tree contains a small number of branching vertices (i.e., vertices of degree at least three), then it is suitable to use the theorem of Doyle and Graver [4] for computing its Wiener index:

Theorem 1.1. Let $T$ be a tree on $n$ vertices. Then

$$
W(T)=\left(\begin{array}{c}
n+1 \\
3
\end{array}\right)-\sum_{v \in V(T)} \sum_{1 \leq i<j<k \leq p} n\left(T_{i}\right) n\left(T_{j}\right) n\left(T_{k}\right),
$$

where $T_{1}, T_{2}, \ldots, T_{p}$ are the components of $T-v$.

Wiener index of the line graph of a tree $T$ can easily be computed from $W(T)$ by using the following result of Buckley [1]:

Theorem 1.2. Let $T$ be a tree on $n$ vertices. Then $W(L(T))=W(T)-\left(\begin{array}{l}n \\ 2\end{array}\right)$.

In [6] (see also [3]) Gutman proposed a problem to find an $n$-vertex graph $G$ whose line graph $L(G)$ has the maximum Wiener index.

Dobrynin and Mel'nikov [3] proposed to estimate the extremal values of the ratio

$$
R_{k}(G)=\frac{W\left(L^{k}(G)\right)}{W(G)}
$$

Notice that

$$
\frac{W\left(L\left(S_{n}\right)\right)}{W\left(S_{n}\right)}=\frac{n-2}{2(n-1)}, \quad \frac{W\left(L\left(P_{n}\right)\right)}{W\left(P_{n}\right)}=\frac{n-2}{n+1}, \quad \text { and } \quad \frac{W\left(L\left(K_{n}\right)\right)}{W\left(K_{n}\right)}=\left(\begin{array}{c}
n-1 \\
2
\end{array}\right) .
$$

In [14], this problem was solved for the minimum in the case $k=1$ :

Theorem 1.3. Among all connected graphs on $n$ vertices, the fraction $R_{1}(G)$ is minimum for the star $S_{n}$.

The problem for the maximum remains open: 
Problem 1.4. Find $n$-vertex graph $G$ with the maximum value of $R_{1}(G)$.

The line graph of $K_{n}$ has the greatest number of edges and the smallest Wiener index, and henceforth, it may attain the maximum value. For higher iterations $k \geq 2$, we expect that the minimum should be at $P_{n}$, as it is the only graph whose line graph decreases in size. Thus, we believe the following holds:

Conjecture 1.5. Let $n$ be a large number and $k \geq 2$. Among all graphs $G$ on $n$ vertices, $W\left(L^{k}(G)\right) / W(G)$ attains the maximum for $K_{n}$, and it attains the minimum for $P_{n}$.

In what follows we support this conjecture for the minimum. In a series of papers $[10,9,12,11,8]$ (see [11, Corollary 1.4]), where the equality $W\left(L^{k}(T)\right)=W(T)$ is solved for trees and $k \geq 3$, the following result was obtained:

Theorem 1.6. Let $T$ be a tree and $k \geq 4$. Then we have

$$
\begin{array}{ll}
W\left(L^{k}(T)\right)=W(T) & \text { if } T \text { is trivial, } \\
W\left(L^{k}(T)\right)<W(T) & \text { if } T \text { is a nontrivial path or the claw } K_{1,3}, \\
W\left(L^{k}(T)\right)>W(T) & \text { otherwise. }
\end{array}
$$

The above result gives an immediate support to Conjecture 1.5:

Corollary 1.7. Let $k \geq 4$. In the class of trees on $n$ vertices, $R_{k}$ attains the minimum value for $P_{n}$.

In this paper we extend the above corollary to the case $k=3$. Let $H$ be a tree on six vertices, two of which have degree 3 and the other four have degree 1 . Recall that two graphs $G_{1}$ and $G_{2}$ are homeomorphic if and only if there is a third graph $F$, such that both $G_{1}$ and $G_{2}$ can be obtained from $F$ by means of edge subdivision. In the proof we will use the following result [9, Corollary 1.6]:

Theorem 1.8. Let $T$ be a tree which is not homeomorphic to a path, claw $K_{1,3}$ or $H$, and let $k \geq 3$. Then $W\left(L^{k}(T)\right)>W(T)$.

By Theorem 1.8, to solve the case $k=3$, it is sufficient to consider the ratios for paths and trees homeomorphic to the claw $K_{1,3}$ and $H$.

Note that $L^{3}\left(P_{n}\right)=P_{n-3}$ if $n \geq 4$, and we have

$$
R_{3}\left(P_{n}\right)=\frac{\left(\begin{array}{c}
n-2 \\
3
\end{array}\right)}{\left(\begin{array}{c}
n+1 \\
3
\end{array}\right)}=\frac{(n-2)(n-3)(n-4)}{(n+1) n(n-1)} .
$$

In Section 2 we prove the following two results:

Theorem 1.9. Let $T$ be a tree on $n$ vertices homeomorphic to $K_{1,3}$. Then

$$
R_{3}(T)>R_{3}\left(P_{n}\right)
$$

Theorem 1.10. Let $T$ be a tree on $n$ vertices homeomorphic to $H$. Then

$$
R_{3}(T)>R_{3}\left(P_{n}\right)
$$

These two results together with Theorem 1.8 and Corollary 1.7 give us the following:

Corollary 1.11. Let $k \geq 3$. Then the path $P_{n}$ attains the minimum value of $R_{k}$ in the class of trees on $n$ vertices. 


\section{Proofs of Theorems 1.9 and 1.10}

Proof of Theorem 1.9. Let $C_{a, b, c}$ be a tree homeomorphic to the claw $K_{1,3}$, such that the paths connecting the vertices of degree 1 with the vertex of degree 3 have lengths $a, b$ and $c$, where $a \geq b \geq c \geq 1$. The tree $C_{a, b, c}$ has exactly $n=a+b+c+1$ vertices, see Figure 1 for $C_{4,3,2}$.

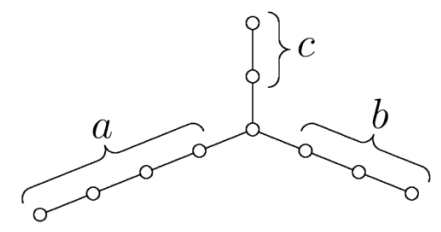

Figure 1: The graph $C_{4,3,2}$.

Further, for $i \in\{1,2,3\}$ let $V_{i}$ be the set of vertices of $V\left(L\left(C_{a, b, c}\right)\right)$ of degree $i$. This naturally splits the problem into four cases according to the size of $V_{1}$.

Denote

$$
\Delta=W\left(L^{3}\left(C_{a, b, c}\right)\right)-W\left(C_{a, b, c}\right)
$$

In [8], the value of $\Delta$ for each of these four cases is evaluated. For the sake of simplicity, let $W_{0}=W\left(C_{a, b, c}\right)$ and $W_{3}=W\left(L^{3}\left(C_{a, b, c}\right)\right)$. Then $\Delta=W_{3}-W_{0}$ and

$$
R_{3}\left(C_{a, b, c}\right)=\frac{W_{3}}{W_{0}}=\frac{W_{0}+\Delta}{W_{0}}=1+\frac{\Delta}{W_{0}} .
$$

By Theorem 1.1 we have

$$
W_{0}=(a+b+c+2)(a+b+c+1)(a+b+c) / 6-a b c .
$$

We prove that when $\left|V\left(C_{a, b, c}\right)\right|=\left|V\left(P_{n}\right)\right|$, that is when $n=a+b+c+1$, then $R_{3}\left(C_{a, b, c}\right)>R_{3}\left(P_{n}\right)$. This inequality is equivalent to

$$
1+\frac{\Delta}{W_{0}}>\frac{(n-2)(n-3)(n-4)}{(n+1) n(n-1)}
$$

and after multiplying by denominators also to

$$
\Delta(n+1) n(n-1)+W_{0}((n+1) n(n-1)-(n-2)(n-3)(n-4))>0 .
$$

Since $3 \geq\left|V_{i}\right| \geq 0$, there are four cases to consider.

Case 1: $\boldsymbol{a}, \boldsymbol{b}, \boldsymbol{c} \geq \mathbf{2}$. That is, $\left|V_{1}\right|=3$. In [8] we have

$$
\Delta=(a+b+c)^{2}-5(a b+a c+b c)+(a+b+c)+21 .
$$

After substituing (2.4) and (2.2) into (2.3), we get that the left-hand side of (2.3) is equal to 
the following expression

$$
\begin{array}{r}
1.5 a b c\left((a-b)^{2}+(a-c)^{2}+(b-c)^{2}\right)+44 a+65 a^{2}+25.5 a^{3}+7 a^{4}+2.5 a^{5}+ \\
44 b+130 a b+66.5 a^{2} b+13 a^{3} b+7.5 a^{4} b+65 b^{2}+66.5 a b^{2}+12 a^{2} b^{2}+10 a^{3} b^{2}+ \\
25.5 b^{3}+13 a b^{3}+10 a^{2} b^{3}+7 b^{4}+7.5 a b^{4}+2.5 b^{5}+44 c+130 a c+66.5 a^{2} c+ \\
13 a^{3} c+7.5 a^{4} c+130 b c+117 a b c+18 a^{2} b c+3 a^{3} b c+66.5 b^{2} c+18 a b^{2} c+ \\
13 b^{3} c+3 a b^{3} c+7.5 b^{4} c+65 c^{2}+66.5 a c^{2}+12 a^{2} c^{2}+10 a^{3} c^{2}+66.5 b c^{2}+18 a b c^{2}+ \\
12 b^{2} c^{2}+10 b^{3} c^{2}+25.5 c^{3}+13 a c^{3}+10 a^{2} c^{3}+13 b c^{3}+3 a b c^{3}+10 b^{2} c^{3}+7 c^{4}+ \\
7.5 a c^{4}+7.5 b c^{4}+2.5 c^{5}
\end{array}
$$

Since $a, b, c \geq 2$, the expression $1.5 a b c\left((a-b)^{2}+(a-c)^{2}+(b-c)^{2}\right)$ and all the isolated terms are nonnegative. Moreover some of the terms, such as $44 a$ for example, are strictly positive. Hence, (2.3) is satisfied, which means that $R_{3}\left(C_{a, b, c}\right)>R_{3}\left(P_{a+b+c+1}\right)$.

Observe that the above long expression was obtained from the left-hand side of (2.3) by subtracting $1.5 a b c\left((a-b)^{2}+(a-c)^{2}+(b-c)^{2}\right)$, which is nonnegative, and then by expanding the difference. Since all the parameters $a, b, c$ are nonnegative, all the coefficients in the expanded expression are positive and at least one of the terms is strictly positive, (2.3) is satisfied. We will use this way of reasoning especially in the proof of Theorem 1.10, where the expanded expressions are extremely long.

Case 2: $\boldsymbol{a}, \boldsymbol{b} \geq \mathbf{2}, \boldsymbol{c}=\mathbf{1}$. That is, $\left|V_{1}\right|=2$. In [8] we have

$$
2 \Delta=(a+b)^{2}-8 a b-5(a+b)+30 .
$$

After substituing (2.5) and (2.2) into (2.3) and expanding the expression, we get that the left-hand side of (2.3) is equal to

$$
\begin{array}{r}
96+170 a+97 a^{2}+32 a^{3}+11 a^{4}+2 a^{5}+170 b+164 a b+43 a^{2} b+11 a^{3} b+6 a^{4} b+ \\
97 b^{2}+43 a b^{2}+8 a^{3} b^{2}+32 b^{3}+11 a b^{3}+8 a^{2} b^{3}+11 b^{4}+6 a b^{4}+2 b^{5}
\end{array}
$$

Hence (2.3) is satisfied and so $R_{3}\left(C_{a, b, 1}\right)>R_{3}\left(P_{a+b+2}\right)$.

Case 3: $\boldsymbol{a} \geq \mathbf{2}, \boldsymbol{b}=\boldsymbol{c}=\mathbf{1}$. That is, $\left|V_{1}\right|=1$. In [8] we have $\Delta=-6 a+6$. After substituing this value of $\Delta$ and (2.2) into (2.3) and expanding the expression, we get that the left-hand side of (2.3) is equal to

$$
1.5 a^{5}+12 a^{4}+26.5 a^{3}+60 a^{2}+300 a+240 .
$$

Hence (2.3) is satisfied and so $R_{3}\left(C_{a, 1,1}\right)>R_{3}\left(P_{a+3}\right)$.

Case 4: $\boldsymbol{a}=\boldsymbol{b}=\boldsymbol{c}=\mathbf{1}$. That is, $\left|V_{1}\right|=0$. In this case $C_{a, b, c}=K_{1,3}$ has 4 vertices and $L^{3}\left(K_{1,3}\right)$ is a cycle of length 3 . Since $W\left(L^{3}\left(P_{4}\right)\right)=0$, we have

$$
R_{3}\left(C_{1,1,1}\right)>0=R_{3}\left(P_{4}\right)
$$

which establishes this small case, and also the proof of the theorem. 
Proof of Theorem 1.10. Denote by $H_{a, b, c, d, e}$ a tree homeomorphic to $H$ defined as follows: In $H_{a, b, c, d, e}$, the two vertices of degree 3 are joined by a path of length $e+1, e \geq 0$. Hence, this path has $e$ vertices of degree 2. Further, at one vertex of degree 3 there start two pendant paths of lengths $a$ and $b$, where $a, b \geq 1$, and at the other vertex of degree 3 there start another two pendant paths of lengths $c$ and $d$, where $c, d \geq 1$. Thus $H_{a, b, c, d, e}$ has $n=a+b+c+d+e+2$ vertices, out of which two have degree 3 , four have degree 1 and the remaining vertices have degree 2, see Figure 2 for $H_{3,3,4,2,2}$. By symmetry, we may assume that $a \geq b, c \geq d$, and $b \geq d$. That is, we assume that the shortest pendant path in $H_{a, b, c, d, e}$ has length $d$.

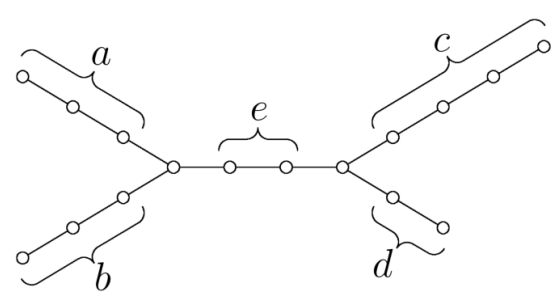

Figure 2: The graph $H_{3,3,4,2,2}$.

We proceed analogously as in the proof of Theorem 1.9. Denote

$$
\Delta=W\left(L^{3}\left(H_{a, b, c, d, e}\right)\right)-W\left(H_{a, b, c, d, e}\right) .
$$

For the sake of simplicity, let $W_{0}=W\left(H_{a, b, c, d, e}\right)$ and $W_{3}=W\left(L^{3}\left(H_{a, b, c, d, e}\right)\right)$. Then $\Delta=W_{3}-W_{0}$ and again

$$
R_{3}\left(H_{a, b, c, d, e}\right)=1+\frac{\Delta}{W_{0}} .
$$

By Theorem 1.1 we have

$$
W_{0}=\left(\begin{array}{c}
a+b+c+d+e+3 \\
3
\end{array}\right)-a b(c+d+e+1)-c d(a+b+e+1) .
$$

If $e=0$, then we have one vertex of degree 4 in $L\left(H_{a, b, c, d, e}\right)$, while if $e \geq 1$, then the greatest degree of a vertex in $L\left(H_{a, b, c, d, e}\right)$ is 3 . Analogously as in [8], by symmetry we distinguish eleven cases. Five cases with at least one of $a, b, c, d$ greater than or equal to 2 have $e \geq 1$, five cases with at least one of $a, b, c, d$ greater than or equal to 2 have $e=0$, and the last case has all $a, b, c, d$ equal to 1 . First we consider the cases with $\Delta>0$.

Claim 1. If $\Delta>0$, then $R_{3}\left(H_{a, b, c, d, e}\right)>R_{3}\left(P_{a+b+c+d+e+2}\right)$.

Proof. Observe that $\left|V\left(H_{a, b, c, d, e}\right)\right|=\left|V\left(P_{a+b+c+d+e+2}\right)\right|$. If $\Delta>0$, then $R_{3}\left(H_{a, b, c, d, e}\right)=1+\frac{\Delta}{W_{0}}>1$. However, $R_{3}\left(P_{n}\right)$ is always smaller than 1 .

By [8], there are 8 cases (out of the 11) for which in [8] it was proved that $\Delta>0$ (we remark that $P$ is used instead of $\Delta$ in [8]). These are the cases:

1. (case 3 in [8]) $a, c \geq 2, b=d=1, e \geq 1$;

2. (case 4 in [8]) $a, b \geq 2, c=d=1, e \geq 1$; 
3. (case 5 in [8]) $a \geq 2, b=c=d=1, e \geq 1$;

4. (case 7 in [8]) $a, b, c \geq 2, d=1, e=0$;

5. (case 8 in [8]) $a, c \geq 2, b=d=1, e=0$;

6. (case 9 in [8]) $a, b \geq 2, c=d=1, e=0$;

7. (case 10 in [8]) $a \geq 2, b=c=d=1, e=0$;

8. (case 11 in [8]) $a=b=c=d=1, e \geq 0$.

By Claim 1 it suffices to consider the remaining three cases.

We proceed analogously as in the proof of Theorem 1.9. Hence, we prove that when $\left|V\left(H_{a, b, c, d, e}\right)\right|=\left|V\left(P_{n}\right)\right|$, that is when $n=a+b+c+d+e+2$, then $R_{3}\left(H_{a, b, c, d, e}\right)>$ $R_{3}\left(P_{n}\right)$. This inequality is equivalent to

$$
1+\frac{\Delta}{W_{0}}>\frac{(n-2)(n-3)(n-4)}{(n+1) n(n-1)}
$$

and after multiplying by denominators also to

$$
\Delta(n+1) n(n-1)+W_{0}((n+1) n(n-1)-(n-2)(n-3)(n-4))>0 .
$$

Now we consider the remaining three cases.

Case 1: $a, b, c, d \geq 2, e \geq 1$. In [8] we have

$$
\begin{aligned}
2 \Delta= & 7(a+b+c+d+e)^{2}-20(a b+a c+a d+b c+b d+c d)-10(a e+b e+c e+d e) \\
& +5(a+b+c+d)+65 e+234 .
\end{aligned}
$$

Denote

$$
\begin{aligned}
D= & 11\left(c d(a-b)^{2}(a+b)+b d(a-c)^{2}(a+c)+a d(b-c)^{2}(b+c)\right. \\
& \left.+b c(a-d)^{2}(a+d)+a c(b-d)^{2}(b+d)+a b(c-d)^{2}(c+d)\right) .
\end{aligned}
$$

Observe that $D \geq 0$. Now substitute (2.9) and (2.7) into the left-hand side of (2.8) and delete $D$. When we expand the resulting expression, all the coefficients will be positive. Since the constant term is 708 , which is strictly positive, (2.8) is satisfied and so $R_{3}\left(H_{a, b, c, d, e}\right)>R_{3}\left(P_{a+b+c+d+e+2}\right)$.

Case 2: $a, b, c \geq 2, d=1, e \geq 1$. From [8] we have

$$
\Delta=3\left(a^{2}+b^{2}+c^{2}+e^{2}\right)-3(a b+a c+b c)+(a e+b e)+2 c e-2(a+b)-c+28 e+97 .
$$

In [8] it was shown that if $e \geq 2$ then $\Delta>0$. By Claim 1 ,

$$
R_{3}\left(H_{a, b, c, 1, e}\right)>R_{3}\left(P_{a+b+c+e+3}\right)
$$

in this subcase, so it suffices to restrict ourselves to $e=1$. For $e=1$ we obtain

$$
\Delta=3\left(a^{2}+b^{2}+c^{2}\right)-3(a b+a c+b c)-a-b+c+128 .
$$


Now substitute (2.10) and (2.7) with $e=1$ into the left-hand side of (2.8). When we expand the resulting expression, all the coefficients will be positive. Since the constant term is 8280, which is strictly positive, (2.8) is satisfied and so $R_{3}\left(H_{a, b, c, 1,1}\right)>R_{3}\left(P_{a+b+c+4}\right)$.

Case 3: $a, b, c, d \geq 2, e=0$. In [8] we have

$$
\Delta=4(a+b+c+d)^{2}-11(a b+a c+a d+b c+b d+c d)+3(a+b+c+d)+137 .
$$

Denote

$$
\begin{aligned}
D= & 10\left(c d(a-b)^{2}(a+b)+b d(a-c)^{2}(a+c)+a d(b-c)^{2}(b+c)\right. \\
& \left.+b c(a-d)^{2}(a+d)+a c(b-d)^{2}(b+d)+a b(c-d)^{2}(c+d)\right) .
\end{aligned}
$$

Observe that $D \geq 0$. Now substitute (2.11) and (2.7) into the left-hand side of (2.8) and delete $D$. When we expand the resulting expression, all the coefficients will be positive. Since the constant term is 828 , which is strictly positive, (2.8) is satisfied and so $R_{3}\left(H_{a, b, c, d, 0}\right)>R_{3}\left(P_{a+b+c+d+2}\right)$. This completes the proof.

\section{References}

[1] F. Buckley, Mean distance in line graphs, in: F. Hoffman, K. B. Reid, R. C. Mullin and R. G. Stanton (eds.), Proceedings of the Twelfth Southeastern Conference on Combinatorics, Graph Theory and Computing, Volume I, Utilitas Mathematica Publishing, Winnipeg, Manitoba, 1981 pp. 153-162, held at Louisiana State University, Baton Rouge, Louisiana, March 2 - 5, 1981.

[2] N. Cohen, D. Dimitrov, R. Krakovski, R. Škrekovski and V. Vukašinović, On Wiener index of graphs and their line graphs, MATCH Commun. Math. Comput. Chem. 64 (2010), 683-698, http://match.pmf.kg.ac.rs/electronic_versions/ Match64/n3/match64n3_683-698.pdf.

[3] A. A. Dobrynin and L. S. Mel'nikov, Wiener index of line graphs, in: I. Gutman and B. Furtula (eds.), Distance in Molecular Graphs - Theory, University of Kragujevac, Kragujevac, volume 12 of Mathematical Chemistry Monographs, pp. 85-121, 2012, http://match.pmf . $\mathrm{kg} \cdot \mathrm{ac} \cdot \mathrm{rs} / \mathrm{mcm} 12 \cdot \mathrm{html}$.

[4] J. K. Doyle and J. E. Graver, Mean distance in a graph, Discrete Math. 7 (1977), 147-154, doi:10.1016/0012-365x(77)90144-3.

[5] R. C. Entringer, D. E. Jackson and D. A. Snyder, Distance in graphs, Czechoslovak Math. J. 26 (1976), 283-296, https://dml.cz/handle/10338.dmlcz/101401.

[6] I. Gutman, Distance of line graphs, Graph Theory Notes N. Y. 31 (1996), 49-52.

[7] I. Gutman and I. G. Zenkevich, Wiener index and vibrational energy, Z. Naturforsch. A 57 (2002), 824-828, http: //www.znaturforsch.com/aa/v57a/s57a0824.pdf.

[8] M. Knor, M. Mačaj, P. Potočnik and R. Škrekovski, Complete solution of equation $W\left(L^{3}(T)\right)=W(T)$ for the Wiener index of iterated line graphs of trees, Discrete Appl. Math. 171 (2014), 90-103, doi:10.1016/j.dam.2014.02.007.

[9] M. Knor, P. Potočnik and R. Škrekovski, On a conjecture about Wiener index in iterated line graphs of trees, Discrete Math. 312 (2012), 1094-1105, doi:10.1016/j.disc.2011.11.023.

[10] M. Knor, P. Potočnik and R. Škrekovski, The Wiener index in iterated line graphs, Discrete Appl. Math. 160 (2012), 2234-2245, doi:10.1016/j.dam.2012.04.021.

[11] M. Knor, P. Potočnik and R. Škrekovski, Wiener index of iterated line graphs of trees homeomorphic to H, Discrete Math. 313 (2013), 1104-1111, doi:10.1016/j.disc.2013.02.005. 
[12] M. Knor, P. Potočnik and R. Škrekovski, Wiener index of iterated line graphs of trees homeomorphic to the claw $K_{1,3}$, Ars Math. Contemp. 6 (2013), 211-219, https:// amc-journal.eu/index.php/amc/article/view/250.

[13] M. Knor and R. Škrekovski, Wiener index of line graphs, in: M. Dehmer and F. Emmert-Streib (eds.), Quantitative Graph Theory: Mathematical Foundations and Applications, CRC Press, Boca Raton, FL, Discrete Mathematics and its Applications (Boca Raton), pp. 279-301, 2015.

[14] M. Knor, R. Škrekovski and A. Tepeh, An inequality between the edge-Wiener index and the Wiener index of a graph, Appl. Math. Comput. 269 (2015), 714-721, doi:10.1016/j.amc.2015. 07.050 .

[15] M. Knor, R. Škrekovski and A. Tepeh, Mathematical aspects of Wiener index, Ars Math. Contemp. 11 (2016), 327-352, https: / / amc-journal.eu/index.php/amc/article/ view/795.

[16] J. Plesník, On the sum of all distances in a graph or digraph, J. Graph Theory 8 (1984), 1-21, doi:10.1002/jgt.3190080102.

[17] H. Wiener, Structural determination of paraffin boiling points, J. Am. Chem. Soc. 69 (1947), 17-20, doi:10.1021/ja01193a005.

[18] K. Xu, M. Liu, K. Ch. Das, I. Gutman and B. Furtula, A survey on graphs extremal with respect to distance-based topological indices, MATCH Commun. Math. Comput. Chem. 71 (2014), 461-508, http://match.pmf.kg.ac.rs/electronic_ versions/Match71/n3/match71n3_461-508.pdf. 